\title{
A new coumarin derivative, IMM-H004, attenuates okadaic acid-induced spatial memory impairment in rats
}

Xiu-yun SONG ${ }^{1}$, Ying-ying WANG ${ }^{1,2}$, Shi-feng $\mathrm{CHU}^{3}$, Jin-feng $\mathrm{HU}^{1}$, Peng-fei YANG ${ }^{1}$, Wei ZUO ${ }^{1}$, Lian-kun SONG ${ }^{1}$, Shuai ZHANG ${ }^{1}$, Nai-hong CHEN $^{1,3, *}$

\author{
${ }^{1}$ State Key Laboratory of Bioactive Substances and Functions of Natural Medicines, Institute of Materia Medica \& Neuroscience \\ Center, Chinese Academy of Medical Sciences and Peking Union Medical College, Beijing 100050, China; ${ }^{2}$ Guangzhou University of \\ Chinese Medicine, Guangzhou 510000, China; ${ }^{3}$ Hunan University of Chinese Medicine, Changsha 410208, China
}

Aim: A novel coumarin derivative 7-hydroxy-5-methoxy-4-methyl-3-(4-methylpiperazin-1-yl)-coumarin (IMM-H004) has shown antiapoptotic, anti-inflammatory and neuroprotective activities. In this study we investigated the effects of IMM-H004 on spatial memory in rats treated with okadaic acid (OKA), which was used to imitate Alzheimer's disease (AD)-like symptoms.

Methods: SD rats were administered IMM-H0O4 $\left(8 \mathrm{mg} \cdot \mathrm{kg}^{-1} \cdot \mathrm{d}^{-1}\right.$, ig) or donepezil (positive control, $1 \mathrm{mg} \cdot \mathrm{kg}^{-1} \cdot \mathrm{d}^{-1}$, ig) for $25 \mathrm{~d}$. On d 8 and 9, OKA (200 ng) was microinjected into the right ventricle. Morris water maze test was used to evaluate the spatial memory impairments. Tau and $\beta$-amyloid $(A \beta)$ pathology in the hippocampus was detected using Western blot and immunohistochemistry. TUNEL staining was used to detect cell apoptosis.

Results: OKA-treated rats showed significant impairments of spatial memory in Morris water maze test, which were largely reversed by administration of IMM-HOO4 or donepezil. Furthermore, OKA-treated rats exhibited significantly increased phosphorylation of tau, deposits of $A \beta$ protein and cell apoptosis in the hippocampus, which were also reversed by administration of IMM-H004 or donepezil. Conclusion: Administration of IMM-HOO4 or donepezil protects rats against OKA-induced spatial memory impairments via attenuating tau or A pathology. Thus, IMM-H0O4 may be developed as a therapeutic agent for the treatment of AD.

Keywords: Alzheimer's disease; coumarin; IMM-H004; donepezil; okadaic acid; memory impairment; Morris water maze test; tau; A $\beta$

Acta Pharmacologica Sinica (2016) 37: 444-452; doi: 10.1038/aps.2015.132; published online 1 Feb 2016

\section{Introduction}

Neurofibrillary tangles (NFTs), which are composed of intracellular filamentous aggregates of hyperphosphorylated tau protein, are one of the pathological hallmarks of Alzheimer's disease (AD). Thus reducing the amount of hyperphosphorylated tau protein might be a therapeutic strategy for treating AD. Previous studies revealed that down-regulation of protein phosphatases or up-regulation of protein kinases are the main causes for tau hyperphosphorylation. Protein phosphatase 2A (PP2A), a major tau phosphatase in the brain, is recognized as a promising candidate that may participate in the regulation of tau phosphorylation in $\mathrm{AD}^{[1]}$.

Okadaic acid (OKA), a selective and potent inhibitor of PP2A, has been reported to induce AD-like hyperphosphory-

\footnotetext{
* To whom correspondence should be addressed.

E-mail chennh@imm.ac.cn

Received 2015-09-01 Accepted 2015-11-20
}

lation of $\mathrm{tau}^{[2]}$. Infusion of OKA into the rat brain causes severe memory impairments that are accompanied by remarkable neuropathological changes, including hippocampal neurodegeneration, a paired helical filament-like phosphorylation of tau, $A \beta$ accumulation and neuronal apoptosis ${ }^{[2-5]}$.

It is known that AD patients and animal models of AD suffer from senile plaques, chronic inflammation and free radical damage. IMM-H004 [7-hydroxy-5-methoxy-4-methyl-3-(4methylpiperazin-1-yl)-coumarin, Figure 1], a novel coumarin compound, has been reported to have neuroprotective effects, especially in neuronal injury resulting from $A \beta$ proteins ( $\beta$-amyloid, the main toxic component of senile plaque in brain of AD patients), lipopolysaccharides (LPS, the revulsive of inflammation) or oxygen glucose deprivation (OGD) ${ }^{[6-8]}$. Furthermore, IMM-H004 also attenuated spatial learning impairments resulting from transient global brain ischemia ${ }^{[9]}$. Therefore, it is important to systematically research the neuroprotective effects of IMM-H004 in AD. The present study 


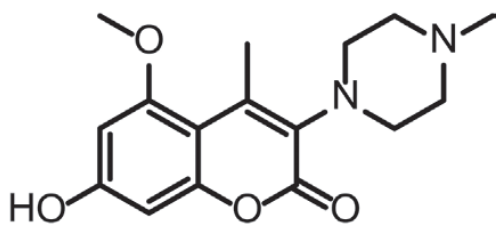

Figure 1. The chemical structure of IMM-H0O4.

was designed to investigate in rats the effects of IMM-H004 on OKA-induced learning and memory deficits and the mechanisms associated with such effects.

\section{Materials and methods Reagents}

The compound IMM-H004 (molecular formula: $\mathrm{C}_{16} \mathrm{H}_{20} \mathrm{O}_{4} \mathrm{~N}_{2}$; molecular weight: 304; chemical purity: $98 \%$ ) was synthesized by Sphinx Scientific Lab Corporation (Tianjin, China). OKA, which was purchased from Sigma-Aldrich (St Louis, MO, USA), was dissolved in saline at a concentration of $40 \mathrm{ng} / \mu \mathrm{L}$. Donepezil was obtained from the National Institutes for Food and Drug Control (Beijing, China).

Anti-tau, anti-p-tau (Ser396) and anti- $\beta$-amyloid primary antibodies were purchased from Abcam (Cambridge, UK). Anti- $\beta$-actin primary antibody was purchased from SigmaAldrich. Anti-rabbit IgG secondary antibody and anti-mouse IgG secondary antibody were obtained from Santa Cruz Biotechnology (Santa Cruz, CA, USA). Enhanced chemiluminescent substrate was obtained from Pierce (Rockford, IL, USA). The TdT-mediated dUTP nick-end labeling (TUNEL) staining kit was purchased from YEASEN Biotechnology Company (Shanghai, China).

\section{Animals}

Adult male Sprague-Dawley (SD) rats $(220 \pm 20$ g, 6-7 weeks old) were obtained from Vital River Laboratories of Beijing (Beijing, China). The rats were housed in a temperatureand light-controlled room $\left(23^{\circ} \mathrm{C}, 12\right.$-h light cycle) and had free access to food and water. All animals were handled in accordance with the standards established in the Guide for the Care and Use of Laboratory Animals published by the Institute of Laboratory Animal Resources of the National Research Council (USA) and approved by the Animal Care Committee of the Peking Union Medical College and the Chinese Academy of Medical Sciences.

\section{Experimental design}

The rats were randomly assigned to 5 groups $(n=12)$. The groups and treatment conditions are detailed in Table 1. The effective doses of OKA, IMM-H004 and donepezil were determined according to our previous studies ${ }^{[5,9]}$. The total duration of the experimental period was 26 days (Figure 2A). The rats were treated with IMM-H004, donepezil or double distilled water as a vehicle by intragastric (ig) administration once daily from $\mathrm{d} 1$ to 25 . On d 8 and 9 , the rats were microinjected with OKA or saline into a lateral cerebral ventricle. One
Table 1. Groups and treatment conditions.

\begin{tabular}{|c|c|c|}
\hline Group treatment & ig (d 1-25) & icv injection (d 8-9) \\
\hline Control & No treatment & No treatment \\
\hline Sham & Vehicle $1(\mathrm{DDW})^{\mathrm{a}}$ & Vehicle $2(5 \mu \mathrm{L} \text { saline })^{b}$ \\
\hline Model & Vehicle $1(\mathrm{DDW})^{\mathrm{a}}$ & $200 \mathrm{ng} / 5 \mu \mathrm{L} \mathrm{OKA^{ \textrm {b } }}$ \\
\hline 1 mg/kg Donepezil & $1 \mathrm{mg} / \mathrm{kg}$ donepezil ${ }^{\mathrm{a}}$ & $200 \mathrm{ng} / 5 \mu \mathrm{L} \mathrm{OKA}^{\mathrm{b}}$ \\
\hline 8 mg/kg IMM-HOO4 & $8 \mathrm{mg} / \mathrm{kg} \mathrm{IMM}-\mathrm{HOO} 4^{\mathrm{a}}$ & $200 \mathrm{ng} / 5 \mu \mathrm{L} \mathrm{OKA}^{\mathrm{b}}$ \\
\hline
\end{tabular}

${ }^{a}$ Administered once daily ig from the 1 st to 25 th day; ${ }^{\text {b Administered icv }}$ injection only one time on d 8 or 9 . DDW, double distilled water.

week after the microinjection, the rats were tested for spatial memory in the Morris water maze for 9 days (Figure 2B, from $\mathrm{d} 17$ to 25). On the last day of the experimental period (d 25) the rats were anesthetized and sacrificed by infusion into the aorta with $0.1 \mathrm{~mol} / \mathrm{L}$ phosphate-buffered saline (PBS, pH 7.4) followed by $4 \%$ paraformaldehyde in PBS (for immunohistochemistry and TUNEL staining) or by decapitation (for Western blot).

\section{Surgery and microinjection}

Surgery was performed and a single intracerebroventricular (icv) injection was administered on either $\mathrm{d} 8$ or 9 . Each rat received only one microinjection. For the microinjections, rats were anesthetized with chloral hydrate $(300 \mathrm{mg} / \mathrm{kg}$, ip) and restrained in a stereotactic apparatus. OKA (200 ng in $5 \mu \mathrm{L}$ saline) or saline $(0.9 \% \mathrm{NaCl}$ for medical use, $5 \mu \mathrm{L})$ was injected into the right lateral cerebral ventricle according to the following coordinates: $0.8 \mathrm{~mm}$ posterior to bregma, 1.5 $\mathrm{mm}$ lateral to the saggital midline, and $3.6 \mathrm{~mm}$ ventral to the dura. Microinjections were performed with a Hamilton micro syringe ${ }^{[10]}$ over a period of $5 \mathrm{~min}(1 \mu \mathrm{L} / \mathrm{min})$. After the microinjection, the needle was kept in place for an additional $5 \mathrm{~min}$

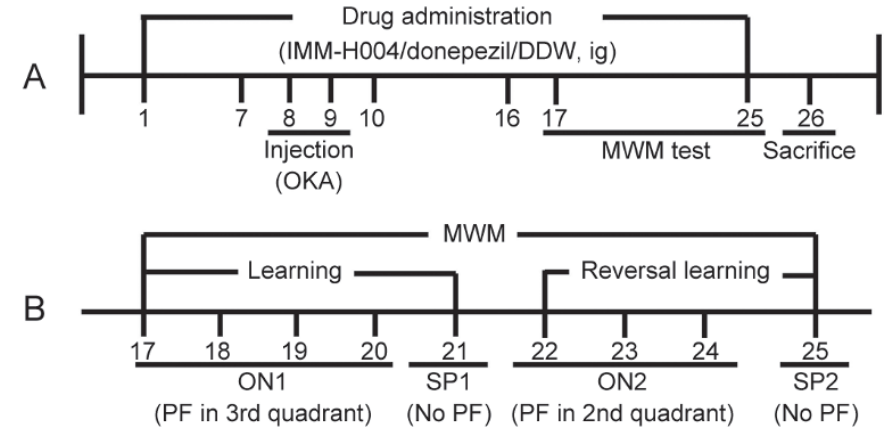

Figure 2. Design of experiments. (A) Rats were administered DDW, IMM-H004 or donepezil for $25 \mathrm{~d}$, and sacrificed on d 26. Rats were microinjected with OKA on d 8 and 9 and tested by Morris water maze (MWM) for $9 \mathrm{~d}$, from d 17 to 25. (B) In the MWM test, rats were trained to search for the platform (PF) below water in the 3rd quadrant from d 17 to 20 (ON1, orientation navigate test 1), which was taken away on d 21 (SP1, spatial probe test 1 ). From d 22 to 24 , rats were trained to search for the new PF in the 2nd quadrant (ON2), which was taken away on d 25 (SP2). 
to allow for diffusion away from the injection site. Finally, the wound was rinsed with penicillin and sutured immediately after the syringe was removed.

\section{Morris water maze test}

The Morris water maze test (Figure 2B) was used to evaluate spatial memory functioning following the treatment. The rats were trained in the Morris water maze by an experimenter who was blind to the treatment schedule (double blind). The water maze apparatus consisted of a metal circular pool (diameter $120 \mathrm{~cm}$, height $80 \mathrm{~cm}$ ), in which a circular Plexiglas platform (diameter $10 \mathrm{~cm}$, height $40 \mathrm{~cm}$ ) was hidden 1-2 $\mathrm{cm}$ below the surface of the water $\left(24 \pm 1^{\circ} \mathrm{C}\right)$. The maze was located in an experimental room that was maintained under stable conditions during the experiment. The task consisted of two consecutive stages: a learning stage and a reversal learning stage. Both stages included two tests, which were the orientation navigation $(\mathrm{ON})$ and spatial probe $(\mathrm{SP})$ tests.

In the first $\mathrm{ON}$ test (ON1) for the learning stage, rats were trained once daily for four consecutive days (from d 17 to 20) to swim to a hidden platform located in the middle of the 3rd quadrant. All tests began at 10:00. At the beginning of a session, each rat was placed in the water, facing the wall of the tank, and given a maximum time of $120 \mathrm{~s}$ to find the hidden platform. Once the rat reached the platform, it was allowed to stay on the platform for $30 \mathrm{~s}$. During each trial, the time required to reach the hidden platform (escape latency) and swim paths were recorded. For the SP1 test, which was performed on $\mathrm{d} 21$, the platform was removed from the tank, and the rats were allowed to swim for $120 \mathrm{~s}$. The number of crossings through the previous platform location were recorded. In the reversal learning stage, rats were forced to find a new platform position ${ }^{[11]}$, which was moved to the middle of 2 nd quadrant. The test procedures and parameters recorded were the same as those for the learning stage. ON2 was performed once daily from d 22 to 24, and SP2 was performed on d 25. Escape latency and crossing times through the platform locations were monitored by a computerized tracking system (Chinese Academy of Medical Sciences, Beijing, China).

\section{Immunohistochemistry}

On d 26, four rats chosen randomly from each group were deeply anesthetized with chloral hydrate $(400 \mathrm{mg} / \mathrm{kg}$, ip) and perfused with $0.1 \mathrm{~mol} / \mathrm{L}$ PBS (pH 7.4) followed by $4 \%$ paraformaldehyde in PBS. Then, the brains were removed, fixed in the same fixative for $24 \mathrm{~h}$, and then dehydrated and subsequently embedded in paraffin blocks. The brain tissues throughout the hippocampus were cut into $4-\mu m$ thick coronal sections for immunohistochemistry.

The paraffin slices were dewaxed with xylol twice to remove paraffin preservative and were hydrated using various concentrations of alcohol $(100 \%, 100 \%, 95 \%, 85 \%$ and $75 \%)$ and distilled water. Then, the slices were incubated for $10 \mathrm{~min}$ at room temperature in $3 \% \mathrm{H}_{2} \mathrm{O}_{2}$ solution to reduce endogenous peroxidase activity and then washed with PBS. The samples were placed in $10 \%$ goat serum for $1 \mathrm{~h}$ at room temperature and incubated overnight at $4{ }^{\circ} \mathrm{C}$ with mouse anti- $\beta$ amyloid or rabbit anti-p-tau primary antibody. Next, the slices were rinsed with PBS and exposed to anti-mouse or anti-rabbit IgG secondary antibody for $1 \mathrm{~h}$ at room temperature. After another rinse in PBS, slices containing $\beta$ amyloid were counterstained with methyl green and then incubated briefly in 3,3'-diaminobenzidine (DAB) substrate (Zhongshan Goldenbridge Biotechnology, Beijing, China) to develop color, whereas slices containing p-tau were incubated briefly in DAB substrate. After a final wash with PBS, the slices were mounted on slides, dehydrated, cleared, and coverslipped with mounting medium.

\section{Western blot analysis}

On d 26, six rats were chosen randomly from each group to obtain samples of hippocampus tissue bilaterally for Western blot analysis. Specifically, the skull was opened to provide access to the brain. Then, cortical tissues were exposed bilaterally from the brain midline using forceps. The hippocampus was then identified and removed. The operation was performed at $4{ }^{\circ} \mathrm{C}$, and the hippocampus tissues were stored at $-80^{\circ} \mathrm{C}$ until use. The hippocampus tissues were homogenized and dissociated with lysis buffer $(50 \mathrm{mmol} / \mathrm{L}$ Tris-HCl, $\mathrm{pH}$ 7.5, $150 \mathrm{mmol} / \mathrm{L} \mathrm{NaCl}$, and protease inhibitor mixture) for $30 \mathrm{~min}$ and then centrifuged at $12000 \times \mathrm{g}$ for $30 \mathrm{~min}$; the supernatants were collected after centrifugation ${ }^{[12]}$. Protein concentrations were determined using the BCA protein assay. Briefly, samples were separated by $10 \%$ SDS-PAGE and then transferred to PVDF membrane. The membranes were blocked with $3 \%$ bovine serum albumin in tris buffer and then incubated overnight at $4{ }^{\circ} \mathrm{C}$ with anti-p-tau (1:2000), anti-tau (1:1000), anti- $\beta$ amyloid (1:500) or anti- $\beta$ actin antibody (1:10000). The membranes were again washed and incubated with secondary antibody (anti-rabbit IgG, 1:5000; anti-mouse IgG, 1:5000) for $1 \mathrm{~h}$ at room temperature. Finally, the bands were marked with enzyme-linked chemiluminescence and were detected using the enhanced chemiluminescence plus detection system (GE Healthcare, Fairfild, CT, USA). The density of each band was quantified using Quantity One analyzer software (Tokyo, Japan).

\section{TUNEL staining}

TUNEL staining was performed for paraffin sections using an in situ cell death detection kit and according to the manufacturer's instructions. After dewaxing and aquation, slices were permeabilized in proteinase $\mathrm{K}(20 \mu \mathrm{g} / \mathrm{mL})$ for $20 \mathrm{~min}$ at room temperature. The slices were then exposed to TdT equilibration buffer, Recombinant TdT Enzyme and Alexa Fluor 488-12-dUTP Labeling Mix for $60 \mathrm{~min}$ at $37^{\circ} \mathrm{C}$. Nuclear staining was identified in cell nuclei with DAPI. DNA breakage was imaged by fluorescence microscopy (Nikon, Tokyo, Japan). Six random non-overlapping sections were viewed and counted under a grid at 100× magnification. All tissue sections were labeled and viewed in a blinded manner and without knowledge of the experimental groups. 


\section{Statistical analysis}

All data are presented as the mean \pm SEM. $P$ values less than 0.05 were considered significant. All analyses were conducted using SPSS 17.0 software (SPSS Inc, Chicago, IL, USA). The escape latencies recorded in the Morris water maze test were analyzed with a repeated measures ANOVA or a multivariate ANOVA. The remaining data were analyzed using a one-way ANOVA with Dunnett's T3 test (unequal variances) or Dunnett's $t$-test (equal variances).

\section{Results}

\section{IMM-H004 attenuated spatial memory impairment}

We evaluated the effects of IMM-H004 and confirmed that the spatial cognition of rats was impaired by the OKA injections by testing the rats' spatial learning abilities in the Morris water maze test (learning stage). In addition, we examined the rats' cognitive flexibility following IMM-H004 administration by relocating the platform in the Morris water maze test (reversal learning stage).

In the learning stage, from d 17 to 20, the escape latencies were recorded. A repeat measures ANOVA showed significant differences at different time points and between groups, but no significant difference was observed in the interaction between Times and Groups (Times: $P<0.001$; Groups: $P=0.002$; Times $\times$ Groups: $P=0.251$ ). In the multivariate ANOVA, we observed a significant difference in latency on d 19 and 20 [d 19: $F(4,53)=5.645, P=0.001 ; d 20: F(4,53)=4.061, P=0.001$, Figure $3 \mathrm{~A}]$. In contrast, the prolonged latency was significantly shortened by IMM-H004 (d 19: $P<0.001$; d 20: $P=0.014$ ) or donepezil (d 19: $P=0.005$; d 20: $P=0.017$ ) administration. Figure $3 \mathrm{~B}$ shows representative diagrams of the search trajectories in the Morris water maze test on d 20. The crossing frequency over the platform location was significantly different among groups on d $21[F(4,50)=3.220, P=0.020$, Figure $3 C]$. However, the decreased times were significantly increased by IMM-H004 administration (IMM-H004: $P=0.011$; donepezil: $P=0.154$ ). In addition, the swimming speed did not vary significantly among groups on d 21 (data not shown).

Comparable performances were also observed during reversal training, in which the hidden platform was moved to a new location. A repeated measures ANOVA for latency in the reversal learning stage revealed similar results as those for the learning stage. A multivariate ANOVA showed a significant difference on d $24[F(4,51)=5.028, P<0.001$, Figure 3D]. However, this was significantly decreased in rats following IMMH004 $(P=0.004)$ or donepezil $(P=0.015)$ administration, which
A

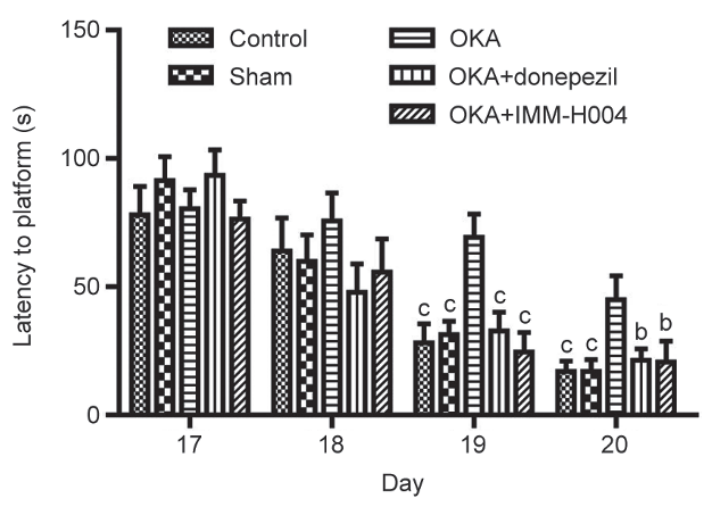

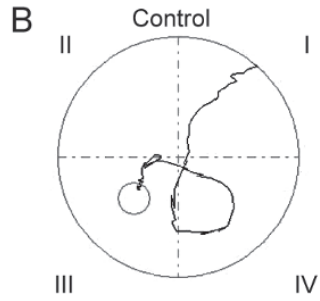
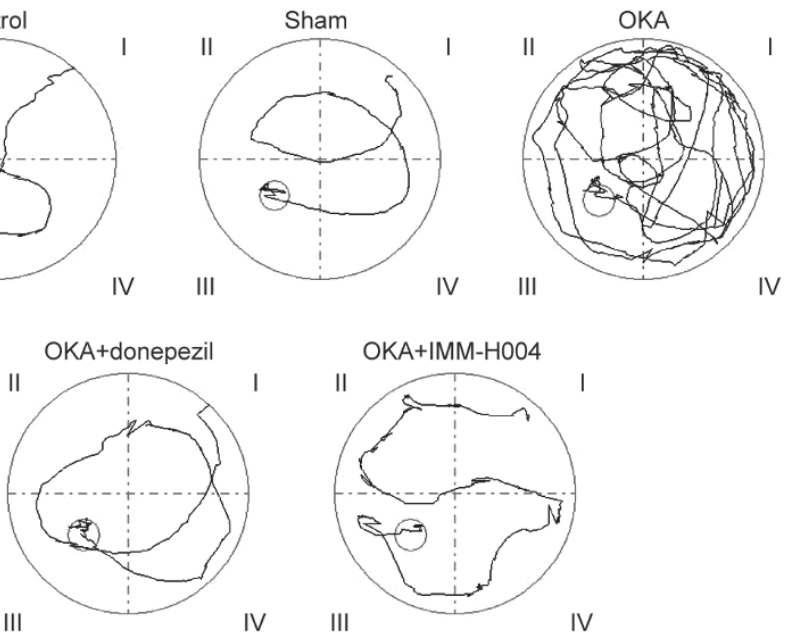

C

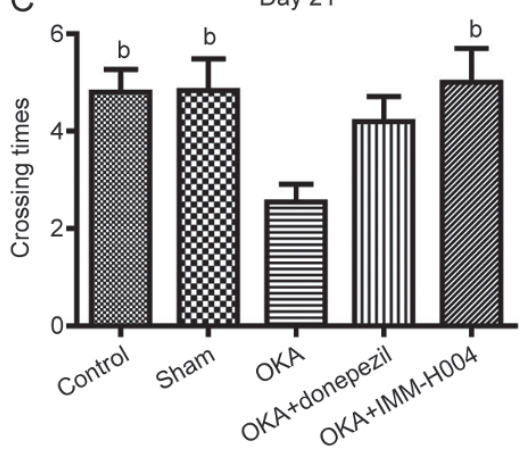

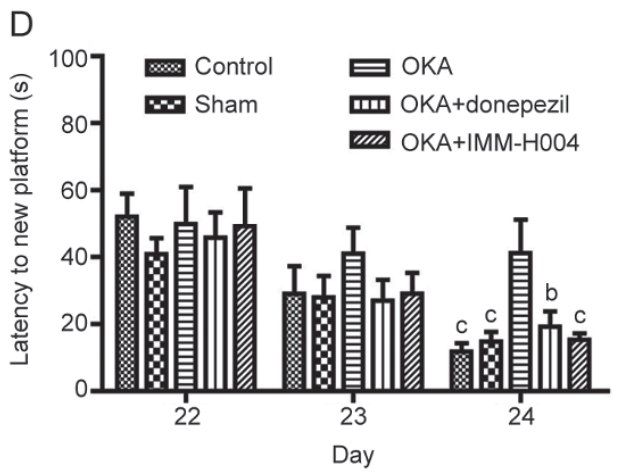

$E$

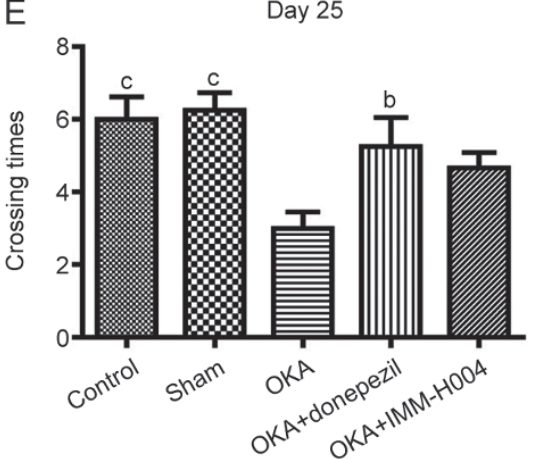

Figure 3. IMM-HOO4 attenuated memory deficits induced by OKA in rats. (A) Escape latency to platform in ON1 test. (B) Paths to the platform during 120-s probe training in ON1 test on d 20. (C) Crossing times through the platform in the SP1 test. (D) Escape latency to platform in the ON2 test. (E) Crossing times through the new platform location in the SP2 test. Mean \pm SEM, $n=11-12 /$ group. ${ }^{b} P<0.05,{ }^{c} P<0.01$ compared with OKA group. 
was consistent with the findings in the learning stage. Furthermore, rats administered donepezil (IMM-H004: $P=0.134$; donepezil: $P=0.025$, Figure $3 E$ ) showed increased times for crossing over the platform on $\mathrm{d} 25$.

Thus, in this experiment, the model rats showed clear deficits in spatial memory consolidation. IMM-H004-induced improvement in these deficits indicates that IMM-H004 plays a role in memory enhancement in a rat model of AD induced by OKA. The effect of limiting spatial memory deficits in reversal learning indicates that IMM-H004 or donepezil could improve cognitive flexibility in rats.

\section{IMM-H004 reversed tau pathology}

The effect of IMM-H004 on tau phosphorylation in the hippocampus was determined by immunohistochemistry and Western blotting. E178 antibody identifying tau Ser396 (the site is aberrantly hyperphosphorylated in NFTs) was used to observe the phosphorylation level of tau. As can be seen in Figure 4A, the OKA group showed weak staining of p-tau; however, overall staining of $p$-tau was stronger in OKAinjected rats compared with that in the sham group, suggesting that tau is hyperphosphorylated after OKA injection. However, IMM-H004 and donepezil administration in rats significantly reversed these alterations. Furthermore, the level of tau phosphorylation, as measured by Western blot analysis $[F(4,25)=10.616, P<0.001$, Figure $4 B, 4 C]$, revealed that phosphorylation of tau was significantly increased compared to that in the sham group $(P<0.001)$, but it was reversed by IMMH004 $(P=0.001)$ or donepezil $(P=0.034)$ administration. Western blot analysis showed that IMM-H004 significantly reduced OKA-induced tau phosphorylation, which was consistent with the results of the immunohistochemistry analysis. These result indicated that IMM-H004 or donepezil could reverse the tau pathology induced by OKA.
A
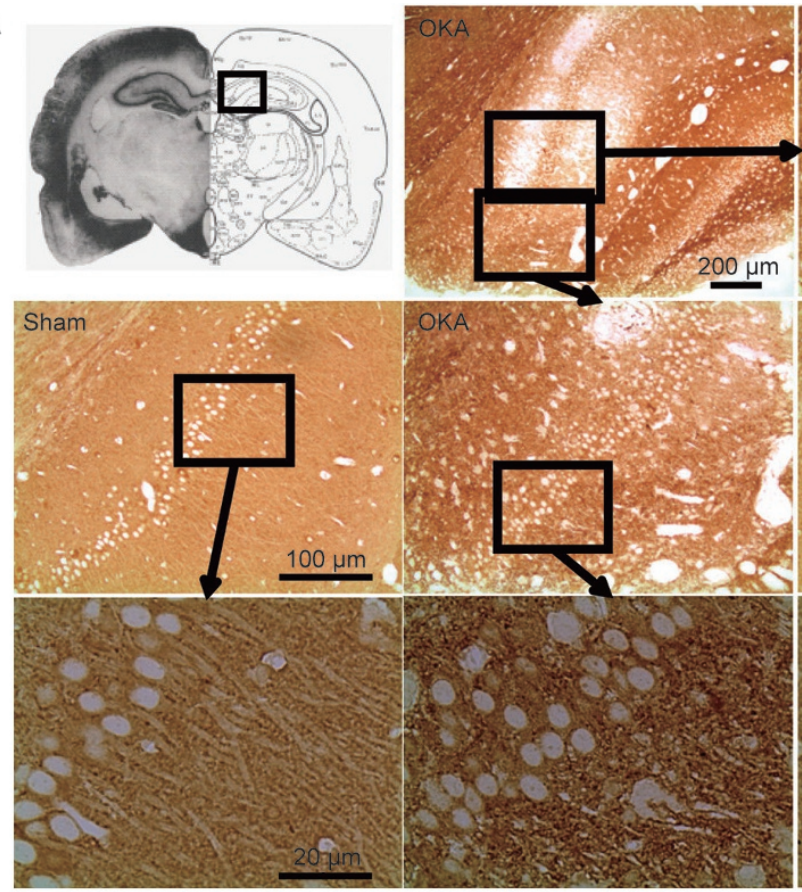

B

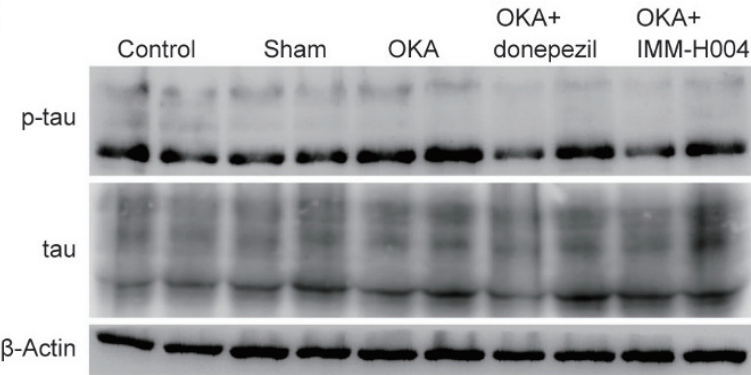

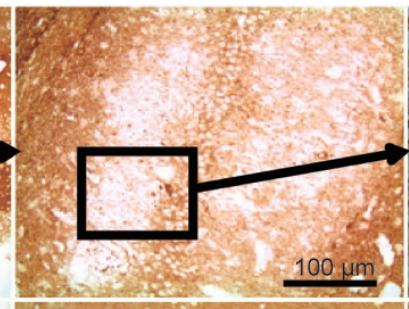
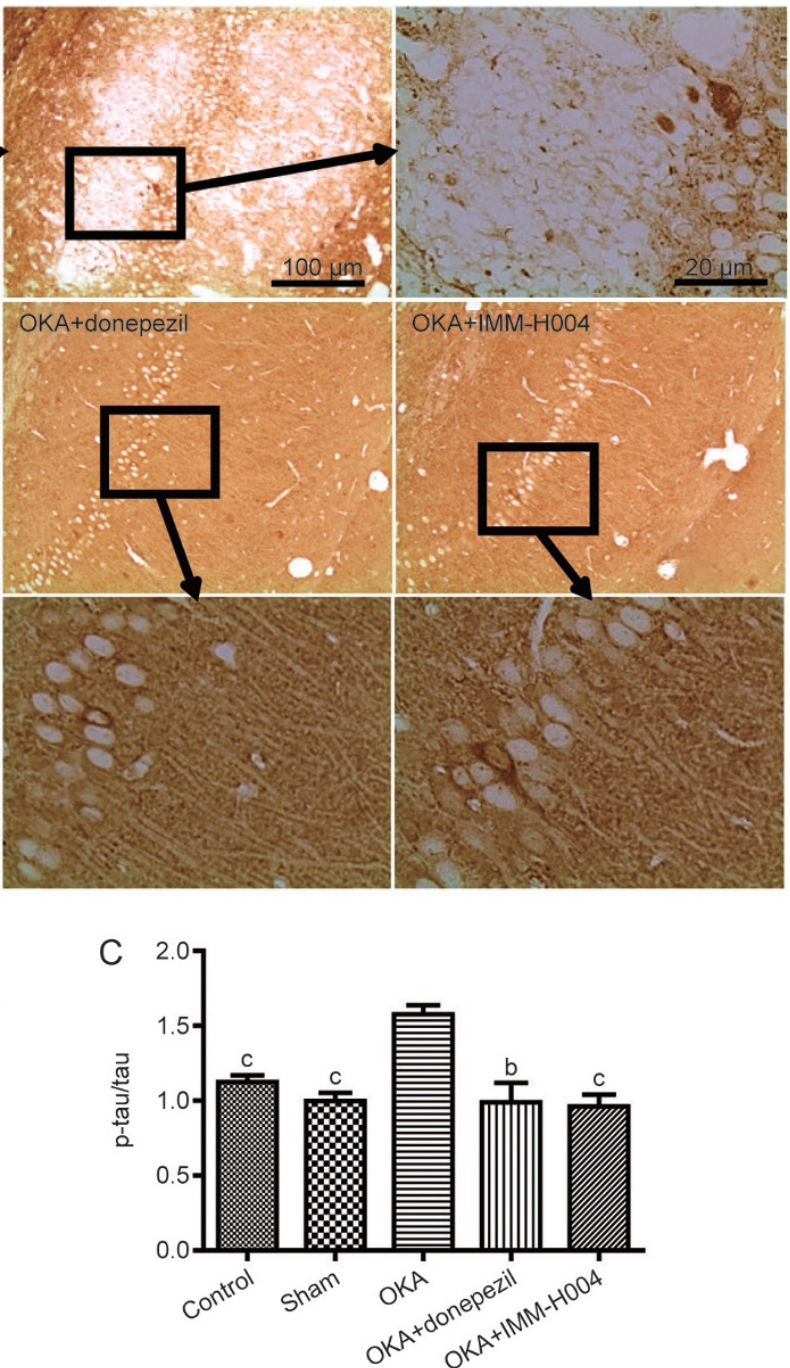

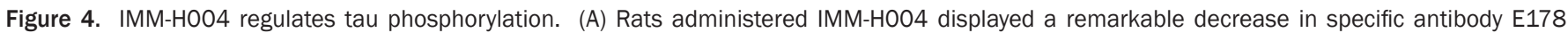

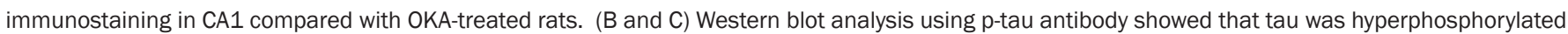

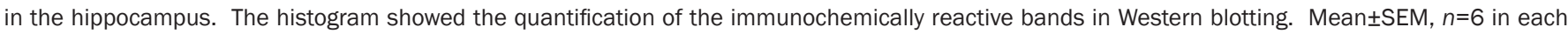
group. One-way ANOVA with Dunnett's T3 test was used for analysis. ${ }^{b} P<0.05,{ }^{c} P<0.01$ compared with OKA group. 


\section{IMM-H004 ameliorated $A \beta$ pathology}

$\beta$-Amyloid antibody (B-4) was used in the immunohistochemistry and Western blot analyses to determine $A \beta$ expression in the hippocampus. As can be seen in Figure $5 \mathrm{~A}, \mathrm{~A} \beta$-positive staining was characterized by brown staining. These images show that $A \beta$ accumulation in the CA1 region increased significantly in the model group. However, both IMM-H004 and donepezil groups showed decreased $A \beta$ expression in the CA1 region. Moreover, the location where strong staining for $A \beta$ was observed was the same location where weak staining for p-tau was observed in the OKA group. The result of the Western blot analysis $[F(4,25)=30.317, P<0.001$, Figure $5 B, 5 C]$ also showed similar results that $A \beta$ expression in the hippocampus increased significantly in the OKA-injected group; and rats administered IMM-H004 $(P<0.001)$ or donepezil $(P=0.034)$ showed a remarkable decrease in $A \beta$ expression compared
A
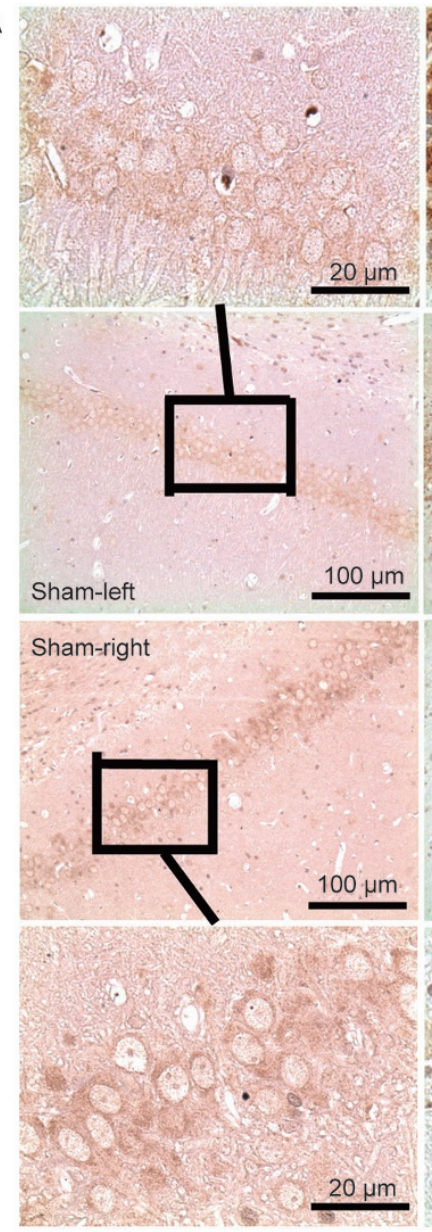
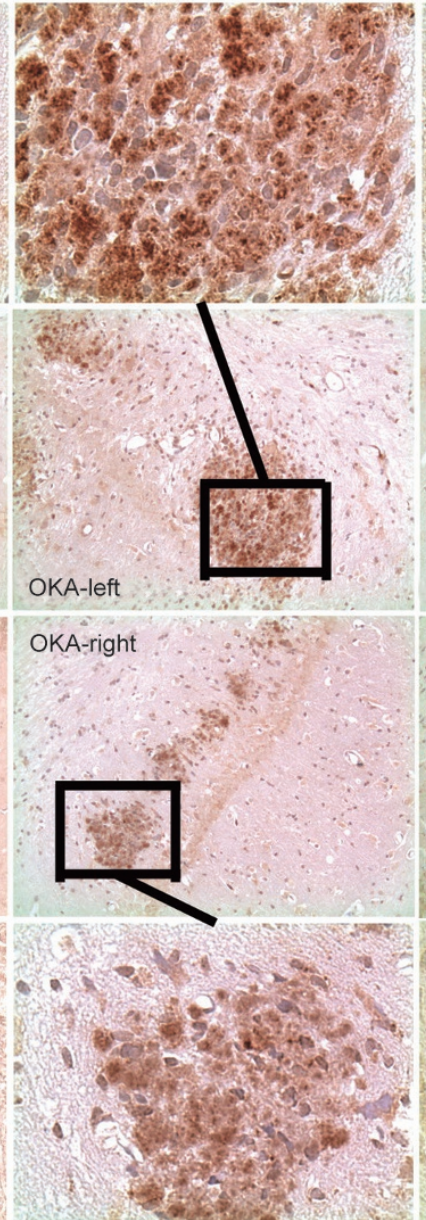

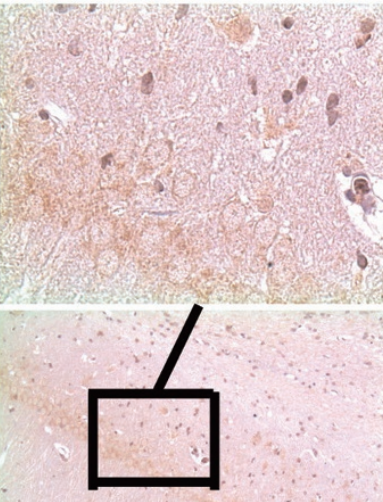

OKA+donepezil-left

OKA+donepezil-right
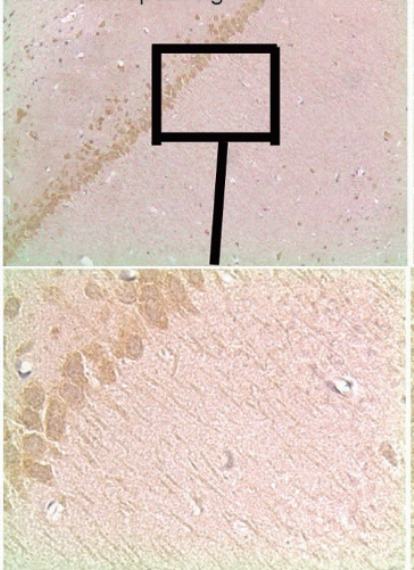
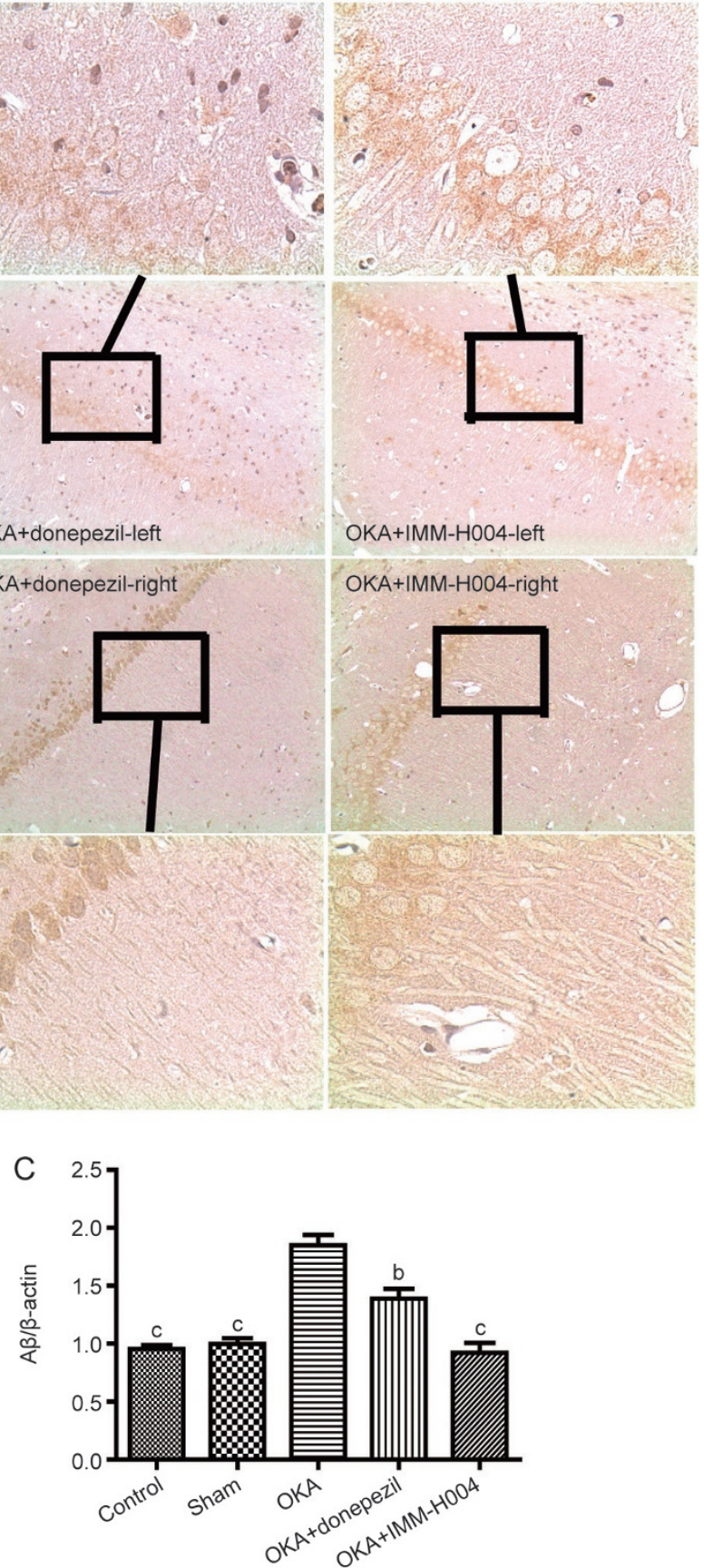

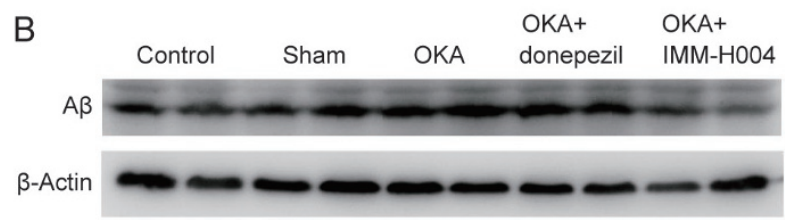

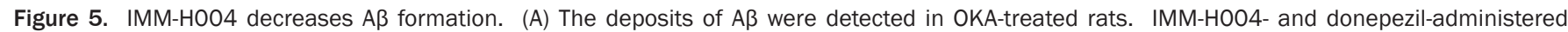

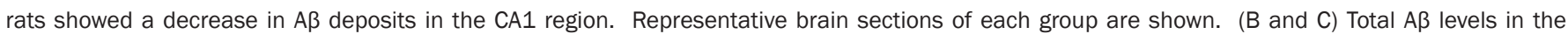

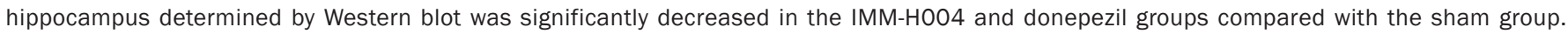
Mean \pm SEM, $n=6$ in each group. One-way ANOVA with Dunnett's T3 test was used for analysis. ${ }^{b} P<0.05$, ${ }^{c} P<0.01$ compared with OKA group. 
to that in the OKA group. Thus, these results suggest that administration of IMM-H004 or donepezil prevented A $\beta$ formation in the hippocampus.

\section{IMM-H004 inhibited apoptosis}

We used TUNEL staining to detect apoptosis in the hippocampus $[F(3,20)=13.484, P<0.001$, Figure $6 \mathrm{~A}, 6 \mathrm{~B}]$. TUNELpositive cells, which were stained with green fluorescence, were not detected in the sham group. However, many cells in the OKA groups were stained with green fluorescence. Nuclei were stained by DAPI with blue fluorescence. Treatment with IMM-H004 $(P=0.001)$ or donepezil $(P=0.007)$ significantly decreased the number of TUNEL-positive cells. This result indicated that IMM-H004 could inhibit apoptosis in the hippocampus. In addition, the location where strong TUNEL staining was identified the same location where weak staining with p-tau and strong staining with $A \beta$ were identified in the OKA group.

\section{Discussion}

Memory impairments have been induced in animal models through microinjection of OKA into the brain ${ }^{[5,13]}$. In the present study, unilateral icv injection of OKA induced spatial memory impairments, which is consistent with previous studies ${ }^{[5]}$. IMM-H004 has been shown to ameliorate spatial learning performance in global ischemia ${ }^{[9]}$, and to inhibit A $\beta$-induced neuronal apoptosis ${ }^{[6,9]}$. Thus, IMM-H004 might be used to improve memory impairment in AD patients. Therefore, further investigation is necessary. In the present study, donepezil, which is a cholinesterase inhibitor that could improve memory in AD animals and patients, was used as a positive control, and we investigated whether IMM-H004 could inhibit OKA-induced memory impairments and its underlying mechanisms.

The Morris water maze test is often used to test the spatial memory ability of animals. The findings of our study demonstrated that rats administered $8 \mathrm{mg} / \mathrm{kg}$ IMM-H004 or $1 \mathrm{mg} / \mathrm{kg}$ donepezil performed similarly as the sham rats did during the learning stage of the Morris water maze test. Thus, IMM-H004 or donepezil treatment reversed spatial memory impairments induced by OKA in rats. The type of learning and memory tested by stable platform paradigms is arguably rare in humans because humans must continually build upon previous memories within a similar context. The Morris water maze task, in which the platform is relocated over time, might challenge learning and memory ${ }^{[14]}$. We therefore used a platform relocation water maze experiment to explore whether IMM-H004 can affect cognitive flexibility in rats that received an icv injection of OKA. The results indicated that IMM-H004 or donepezil improved the rats' performance in response to challenges in learning and memory by shortening the escape latency and increasing their crossing frequency in the right quadrant, where the platform was previously located. Hence, IMM-H004 improved cognitive flexibility and may attenuate spatial memory injury.

Tau is a phosphoprotein that plays a crucial role in microtubule assembly and stability ${ }^{[15]}$. The aberrantly hyperphosphorylated tau in the AD brain leads to disruption of the neuronal cytoskeleton and axonal transport ${ }^{[16]}$. Additionally, abnormal hyperphosphorylation also makes tau resistant to proteolytic
A

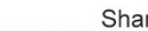

Sham

OKA

OKA+donepezil

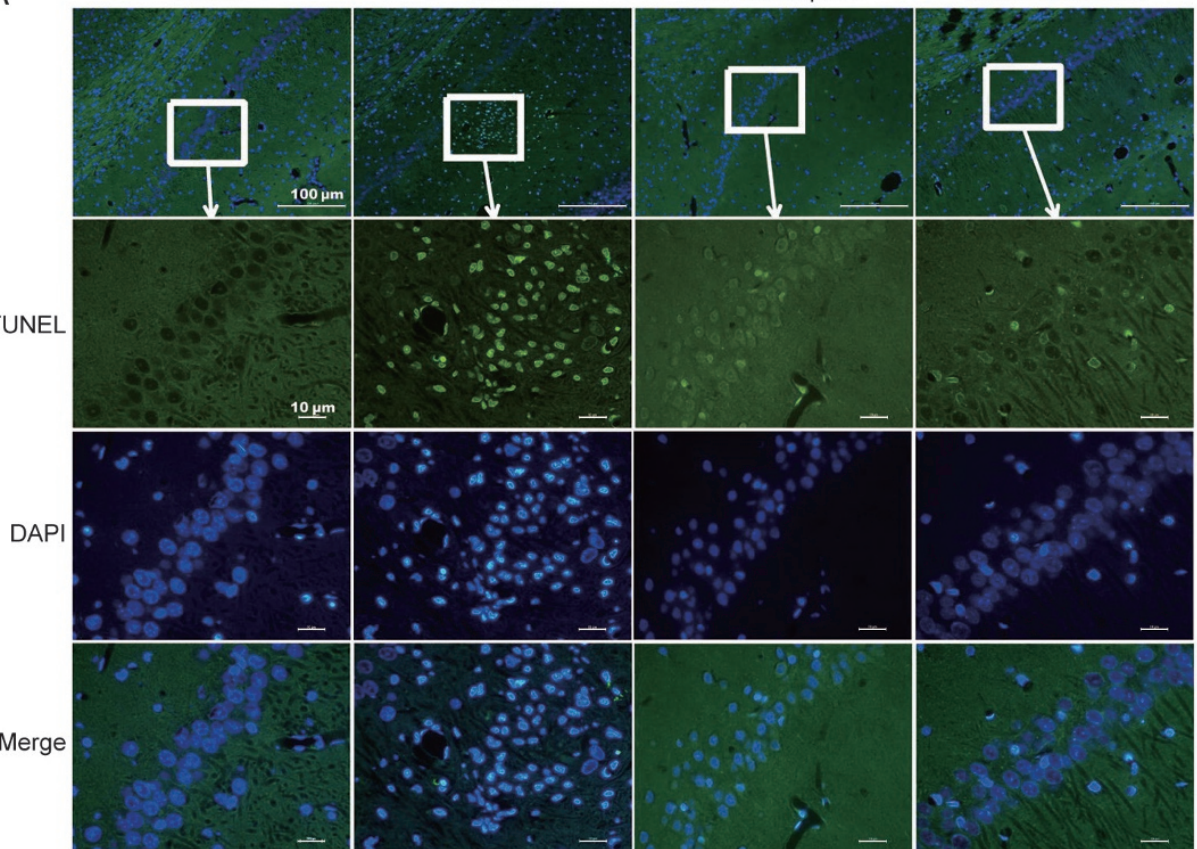

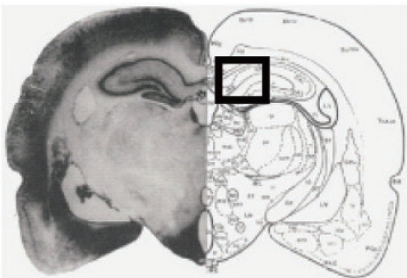

B

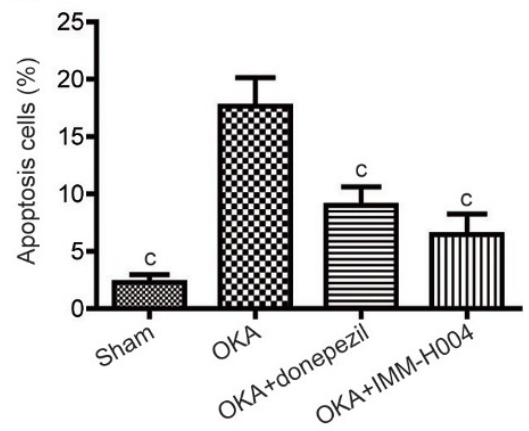

Figure 6. IMM-HOO4 inhibits cell apoptosis. (A) Representative images of TUNEL staining in the CA1 region of the hippocampus. (B) The number of apoptotic cells in hippocampus was analyzed. Mean \pm SEM, $n=6$. One-way ANOVA with Dunnett's $t$-test was used for analysis. ${ }^{\circ} P<0.01$ vs $0 K A$ group. 
degradation, which leads to its gradual accumulation in the cell and promotes the formation of NFTs ${ }^{[17]}$. In this study, the immunohistochemistry and Western blot analyses showed that OKA induced unusual tau hyperphosphorylation in the hippocampus. However, IMM-H004 administration decreased the level of p-tau, which contributed to an improved biological activity of tau in promoting the assembly and maintaining the stability of the microtubules, as well as preventing the formation of NFTs.

Senile plaques that induce extracellular $A \beta$ aggregation is another pathological characteristic of $\mathrm{AD}^{[18]}$. The neurotoxicity of $\mathrm{A} \beta$ has been shown in vitro ${ }^{[6,19]}$ and in vivo ${ }^{[20,21]}$. $\mathrm{A} \beta$, tau and their associated signaling pathways represent important therapeutic targets for AD. Furthermore, A $\beta$ inhibits degradation of hyperphosphorylated tau by proteasomes ${ }^{[2]}$. However, the relationship between tau and $A \beta$, and which one of these two proteins is the primary cause of $A D$, remain unclear. Currently, a growing body of work supports the notion that $\mathrm{A} \beta$ regulates the phosphorylation of tau to accelerate NFT formation $^{[18]}$. Additionally, the hyperphosphorylation of tau protein increases the accumulation of $A \beta$ and decreases its degradation $^{[4,23]}$. In the present study, we found that icv injection of OKA increased $A \beta$ expression, which is consistent with previous reports ${ }^{[3,4]}$. Both immunohistochemistry and Western blot results showed that treatment with IMM-H004 downregulated OKA-induced $A \beta$ formation in the hippocampus.

OKA (200 ng) icv microinjection induced spatial memory impairments, as well as apoptosis in the CA1 region of the hippocampus. IMM-H004 or donepezil administration significantly attenuated the OKA-induced increase in the number of apoptotic cells. Therefore, the beneficial effects of IMM-H004 on OKA-induced spatial memory impairments in rats may be subsequent to its attenuating effects on OKA-induced apoptosis in hippocampal cells.

The hippocampus plays a critical role in learning and memory, and the most important parts for learning and memory in the hippocampal regions are the CA1, CA3 and dentate gyrus regions ${ }^{[24]}$. Our results demonstrated that an icv injection of OKA increased the unusual phosphorylation of tau in the hippocampus. Moreover, OKA treatment also led to a significant accumulation of $A \beta$ in CA1. These results indicated that the changes observed in the CA1 region of the hippocampus may contribute to the decline in memory observed in AD. Therefore, IMM-H004 may prevent spatial learning and memory decline resulting from an icv injection of OKA by decreasing $\mathrm{A} \beta$ formation and the level of $\mathrm{p}$-tau in the CA1 region.

As Figure 4A, 5A, and 6A showed, the locations for weak p-tau staining were the same locations for strong $A \beta$ and apoptotic cell staining in the OKA group. Therefore, OKA (200 ng) icv injection first induced tau hyperphosphorylation, which further increased the $\mathrm{A} \beta$ burden and apoptosis.

In this study, the positive control, which was the cholinesterase inhibitor donepezil, could also decrease hyperphosphorylation of tau, $\beta$-amyloid plaques and apoptosis. These results were similar to those obtained with IMM-H004. Kamat et al indicated that donepezil could improve OKA-induced memory damage by increasing PP2A expression ${ }^{[25]}$, which decreased hyperphosphorylation of tau, or by increasing cholinergic function ${ }^{[26]}$. Noh et al ${ }^{[27]}$ showed that donepezil improved A $\beta 1-42$-induced neurotoxicity through activation of PP2A, which could decrease hyperphosphorylation of tau. Furthermore, donepezil could attenuate $A \beta$-associated mitochondrial dysfunction and reduce mitochondrial $A \beta$ accumulation in vivo and in vitro, which might differ from its previously reported effect on $\mathrm{AChE}^{[28]}$. Our previous study ${ }^{[5]}$ indicated that donepezil could ameliorate OKA-induced hyperphosphorylation of tau and $A \beta$ accumulation. Therefore, donepezil improved OKA-induced neurotoxicity possibly through PP2A activation or by improving cholinergic functioning. However, the upstream target of IMM-H004 that resulted in reversed tau and $A \beta$ pathology is still unclear and requires further investigation.

In conclusion (Figure 7), unilateral icv microinjection of OKA significantly induced spatial memory impairments, caused hyperphosphorylation of tau protein, and increased the $\mathrm{A} \beta$ burden and apoptosis in the hippocampus of rats. Administration of IMM-H004 effectively attenuated OKAinduced apoptosis and spatial memory impairments. These effects were likely mediated through two possible mechanisms: (1) IMM-H004 stabilized the tau pathology, which further decreased the A $\beta$ content; and (2) IMM-H004 attenuated $\mathrm{A} \beta$ accumulation. These findings suggest that IMM-H004 may have therapeutic effects in the treatment of cognitive impairments and neuropathological changes that occur in AD and other neurodegenerative diseases.

\section{Acknowledgements}

This work was supported by the National Natural Science Foundation of China (No 81274122, 81173578, 81373997, 81473570, and U1402221), the National Mega-project for Innovative Drugs (№ 2012ZX09103101-006, 2012ZX09301002001-002, and 2012ZX09301002-004), the Program for Changjiang Scholars and Innovative Research Team in University (PCSIRT) (No IRT1007), the National High-Tech R\&D Pro-

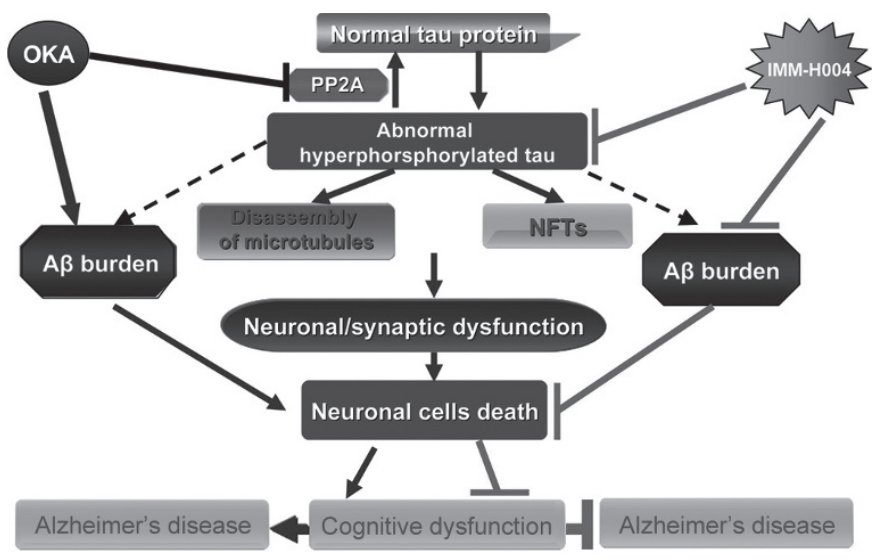

Figure 7. The mechanisms under the beneficial effects of IMM-HOO4 on OKA-induced cognitive dysfunction. 
gramme (863 Program) (№ 2012AA020303), the Specialized Research Fund for the Doctoral Program of Higher Education of China (№ 20121106130001), Beijing Natural Science Foundation (№ 7131013) and Beijing Key Laboratory of New Drug Mechanisms and Pharmacological Evaluation Study (№ BZ0150).

\section{Author contribution}

Nai-hong CHEN is the chief for the study; Xiu-yun SONG and Jin-feng HU conceived this study; Xiu-yun SONG and Yingying WANG performed the main experiments; Xiu-yun SONG and Peng-fei YANG analyzed the data and wrote the paper; Wei ZUO, Lian-Kun SONG and Shuai ZHANG assisted in the experiments; Shi-feng CHU assisted in conceptual writing. All authors have read and approved the final manuscript.

\section{References}

1 Gong CX, Lidsky T, Wegiel J, Zuck L, Grundke-lqbal I, Iqbal K. Phosphorylation of microtubule-associated protein tau is regulated by protein phosphatase $2 \mathrm{~A}$ in mammalian brain. Implications for neurofibrillary degeneration in Alzheimer's disease. J Biol Chem 2000; 275: 5535-44.

2 Kamat PK, Rai S, Swarnkar S, Shukar R, Nath C. Molecular and cellular mechanism of okadaic acid (OKA)-induced neurotoxicity: a novel tool for Alzheimer's disease therapeutic application. Mol Neurobiol 2014; 50: 852-65.

3 Arendt T, Holzer M, Fruth R, Bruckner MK, Gartner U. Paired helical filament-like phosphorylation of tau, deposition of beta/A4-amyloid and memory impairment in rat induced by chronic inhibition of phosphatase 1 and 2A. Neuroscience 1995; 69: 691-8.

4 Arendt T, Holzer M, Fruth R, Bruckner MK, Gartner U. Phosphorylation of tau, Abeta-formation, and apoptosis after in vivo inhibition of PP-1 and PP-2A. Neurobiol Aging 1998; 19: 3-13.

5 Song XY, Hu JF, Chu SF, Zhang Z, Xu S, Yuan YH, et al. Ginsenoside Rg1 attenuates okadaic acid induced spatial memory impairment by the GSK $3 \beta /$ tau signaling pathway and the $A \beta$ formation prevention in rats. Eur J Pharmacol 2013; 710: 29-38.

6 Song XY, Hu JF, Sun MN, Li ZP, Wu DH, Ji HJ, et al. IMM-H004, a novel coumarin derivative compound, protects against amyloid beta-induced neurotoxicity through a mitochondrial-dependent pathway. Neuroscience 2013; 242: 28-38.

7 Song XY, Hu JF, Sun MN, Li ZP, Zhu ZX, Song LK, et al. IMM-H004, a novel coumarin derivative compound, attenuates the production of inflammatory mediatory mediators in lipopolysaccharide-activated BV2 microglia. Brain Res Bull 2014; 106: 30-8.

8 Ji HJ, Wang DM, Hu JF, Sun MN, Li G, Li ZP, et al. IMM-H004, a novel courmarin derivative, protects against oxygen- and glucose-deprivation/ restoration-induced apoptosis in PC12 cells. Eur J Pharmacol 2014; 723: 259-66.

9 Zuo W, Zhang W, Han N, Chen NH. Compound IMM-H004, a novel coumarin derivative, protects against CA1 cell loss and spatial learning impairments resulting from transient global ischemia. CNS Neurosci Ther 2015; 21: 280-8.

10 Paxinos G, Watson CR, Emson PC. AChE-stained horizontal sections of the rat brain in stereotaxic coordinates. J Neurosci Methods 1980; 3: $129-49$.
11 Vorhees CV, Williams MT. Morris water maze: procedures for assessing spatial and related forms of learning and memory. Nat Protoc 2002; 1: 848-58.

12 Gao Y, Chu SF, Li JP, Zhang Z, Yan JQ, Wen ZL, et al. Protopanaxtriol protects against 3-nitropropionic acid-induced oxidative stress in a rat model of Huntington's disease. Acta Pharmacol Sin 2015; 36: 311-22.

13 Kamat PK, Tota S, Saxena G, Shukla R, Nath C. Okadaic acid (ICV) induced memory impairment in rats: a suitable experimental model to test anti-dementia activity. Brain Res 2010; 1309: 66-74.

14 Saab BJ, Saab AM, Roder JC. Statistical and theoretical considerations for the platform re-location water maze. J Neurosci Methods 2011; 198: 44-52.

15 Iqbal K, Alonso Adel C, Grundke-lqbal I. Cytosolic abnormally hyperphosphorylated tau but not paired helical filaments sequester normal MAPs and inhibit microtubule assembly. J Alzheimers Dis 2008; 14 : 365-70.

16 Das V, Miller JH. Microtubule stabilization by peloruside A and paclitaxel rescues degenerating neurons from okadaic acid-induced tau phosphorylation. Eur J Neurosci 2012; 35: 1705-17.

17 Merrick SE, Trojanowski JQ, Lee VM. Selective destruction of stable microtubules and axons by inhibitors of protein serine/threonine phosphatases in cultured human neurons. J Neurosci 1997; 17: 5726-37.

18 Huang HC, Jiang ZF. Accumulated amyloid-beta peptide and hyperphosphorylated tau protein: relationship and links in Alzheimer's disease. J Alzheimers dis 2009; 16: 15-27.

19 Sui HJ, Zhang LL, Liu Z, Jin Y. Atorvastatin prevents $A \beta$ oligomerinduced neurotoxicity in cultured rat hippocampal neurons by inhibiting Tau cleavage. Acta Pharmacol Sin 2015; 36: 553-64.

20 AngelovaPP, AbramovAY. Interaction of neurons and astrocytes underlies the mechanism of $A \beta$-induced neurotoxicity. BiochemSoc Trans 2014; 42: 1286-90.

21 Billings LM, Oddo S, Green KN, McGaugh JL, LaFerla FM. Intraneuronal Abeta causes the onset of early Alzheimer's disease-related cognitive deficits in transgenic mice. Neuron 2005; 45: 675-88.

22 Wang Y, Mandelkow E. Degradation of tau protein by autophagy and proteasomal pathways. Biochem Soc trans 2012; 40: 644-52.

23 Desikan RS, McEvoy LK, Thompson WK, Holland D, Brewer JB, Aisen PS, et al. Amyloid-beta--associated clinical decline occurs only in the presence of elevated P-tau. Arch Neurol 2012; 69: 709-13.

24 Knierim JJ, Lee I, Harqreaves EL. Hippocampal place cells: parallel input streams, subregional processing, and implications for episodic memory. Hippocampus 2006; 16: 755-64.

25 Kamat PK, Rai S, Swarnkar S, Shukla R, Ali S, Najmi AK, et al. Okadaic acid-induced Tau phosphorylation in rat brain: Role of NMDA receptor. Neuroscience 2013; 238: 97-113.

26 Kamat PK, Tota S, Rai S, Shukla R, Ali S, Najmi AK, et al. Okadaic acid induced neurotoxicity leads to central cholinergic dysfunction in rats. Eur J Pharmacol 2012; 690: 90-8.

27 Noh MY, Koh SH, Kim SM, Maurice T, Ku SK, Kim SH. Neuroprotective effects of donepezil against $A \beta 42$-induced neuronal toxicity are mediated through not only enhancing PP2A activity but also regulating GSK-3 $\beta$ and nAChRs activity. J Neurochem 2013; 127: 562-74.

28 Ye CY, Lei Y, Tang XC, Zhang HY. Donepezil attenuates A $\beta$ associated mitochondrial dysfunction and reduces mitochondrial $A \beta$ accumulation in vivo and in vitro. Neuropharmacology 2015; 95 : 29-36. 